\title{
The biological efficacy of the transfer function
}

\author{
H. WILLIAM COLLINS and FREDERICK J. BREMNER \\ Trinity University, San Antonio, Texas
}

\begin{abstract}
The most popular neural network strategy is back propagation. This strategy initiated general interest in neural networks among researchers. While back propagation can solve nonlinear problems, it is considered to be a poor example of neuron functioning. Recently, Gardner (1993) has made a strong case for a back propagating phenomenon in networks of living neurons. In this paper, we present a few simple computational examples that investigate another component of the typical back propagation network. The effects of varying transfer functions are illustrated along with the resulting variations in possible synaptic weights. Graphic presentations in 3-D space of the relationship between transfer functions and synaptic weights suggest neural analogies of cell-firing rate and network control.
\end{abstract}

The back propagation strategy used in neural modeling is generally considered to be a poor approximation of the real biological events that change neurons during learning (Harvey, 1994; MacGregor, 1993). In particular, the strategy of retroflexively affecting a presynaptic membrane, which is the sine qua non of back propagation, is contrary to long-standing biological evidence. Nevertheless, evidence elegantly presented by Gardner (1993) makes a strong case for a retrograde effect of the postsynaptic cell membrane on the presynaptic membrane in a way analogous to back propagation. In the light of this new evidence, it seems that back propagation is more neuromorphic than was originally believed.

Gardner's (1993) insight into the neural analogy of back propagation has prompted us to reconsider the neuromorphology of other mathematical components used by the back propagation strategy. This paper focuses on the transfer function and the relationship of synaptic weights generated via back propagation. The transfer function bounds the output of the simulated neuron by adjusting the activation function. In the typical back propagation computer program, the activation function is a multiplication of the incoming signal by the potency of the synapses (the weight, $w_{j i}$ ), and the addition of a value generated by a limiting function (the threshold). The resultant of these equations is then squashed by the transfer function, which sets upper, lower, and rate-of-change limits on the transmitted signal (Mpitsos, Burton, \& Creech, 1988). Similarly, the biological neuron is bounded by its rate of firing, changes in firing rate, and its pattern. Although the maximum firing rate of a neuron is considered to be $1000 \mathrm{~Hz}$, few neurons, if any, fire at their maximum rate (Bremner \& Denham, 1993; Hinton \& Anderson, 1981).

The purpose of this experiment was to investigate two transfer functions representing the neuron's manipulation of the incoming signal to produce the biological out-

Correspondence should be addressed to F. J. Bremner, Department of Psychology, Trinity University, 715 Stadium Dr., San Antonio, TX 78212-7200. put. One of these transfer functions was linear and the other was sigmoid. These two transfer functions were applied to two chaotic data sets, one in which the task was to output the exact input (identity function task) and the other in which it was to predict the next value of a chaotic array (predictive task). The hypothesis was that if the task is linear, a linear transfer function would be best, and if the task is nonlinear, the sigmoid transfer function would be best.

\section{METHOD}

\section{Apparatus}

For our investigation we programmed a standard three-layer network in MATLAB Version 4.0 (The Math Works, Inc., Natick, MA). This package is widely used for numerical computations in the scientific and engineering community because of its matrix operations and extensive data presentation capabilities. MATLAB can be programmed in a high-level language similar to BASIC or FORTRAN, but without the cumbersome looping structures that usually exist for matrix operations. This gave us code that was short and concise, but flexible enough to experiment with variations in network representation.

The network simulations were made using a Dell 433 (80486DX processor running at $33 \mathrm{MHz}$ ) microcomputer operating under Windows 3.1. Training times for the investigated systems varied from $10 \mathrm{sec}$ to $1.5 \mathrm{~min}$, depending on the size and convergence properties of the network.

\section{Procedure}

The simple three-layered network was trained using different but similar transfer functions. The network had a single input neuron and a single output neuron, with three neurons in the hidden layer. We trained this network to do the identity function task (if $x$ was the input, $\mathrm{x}$ was the output) of a data set generated by the logistic equation with $K$ equal to 3.95 (Bremner \& Yost, 1992). The transfer functions tested were (1) the standard sigmoid and (2) a linear function bounded by 0 and 1 . Our system used a sigmoid transfer function similar to that reported earlier by Mpitsos et al. (1988). The bounded linear function is similar to the sigmoid function in that both have the same slope at their midpoint value $[(\max +\min ) / 4)]$; however, the linear function approaches its limit ( 0 or 1$)$ more quickly than does the sigmoid (Figure 1). The network was trained by randomly selecting all initial weights and threshold values, then using the back propagation method to adjust both. We used the same 
A. Sigmoid Transfer Function

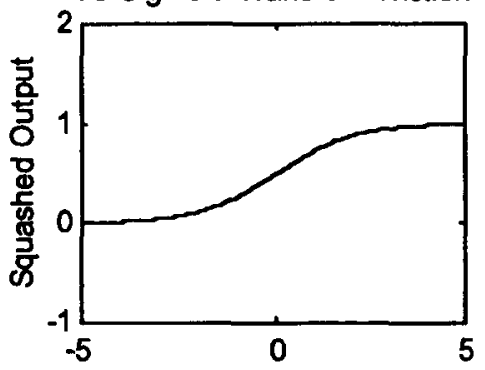

C. Trained Network using Sigmoid

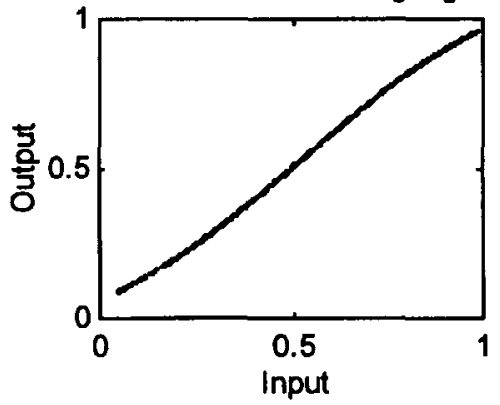

B. Bounded Linear Function

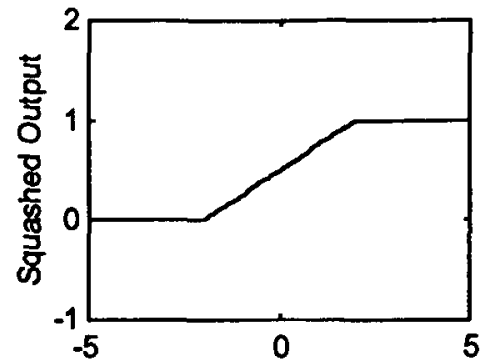

D. Trained Network using Linear

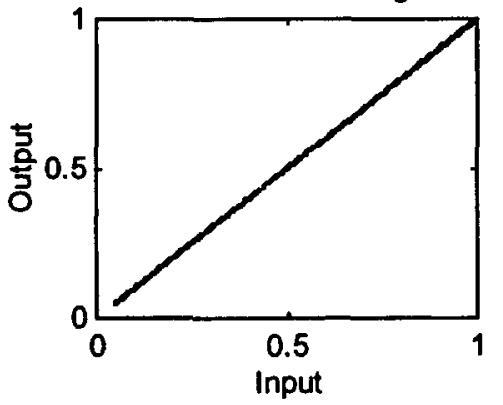

Figure 1. Results of the network when trained to transmit the chaotic input (the identity task). A perfectly trained network should display a diagonal line from $(0,0)$ to $(1,1)$ as in Panel $D$. Therefore, the linear transfer shown in $B$ gave the best result on the identity task.

A. Trained Network using Sigmoid

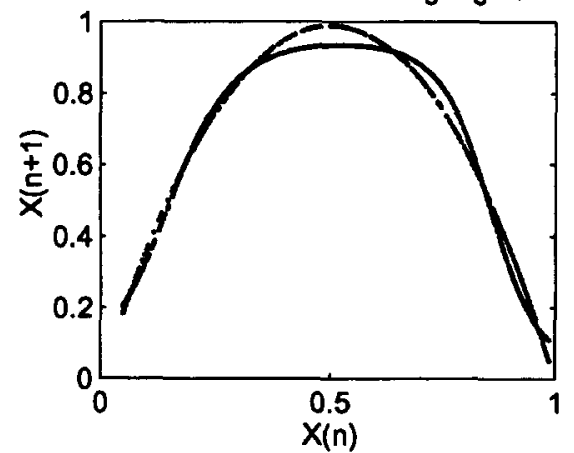

B. Trained Network using Linear

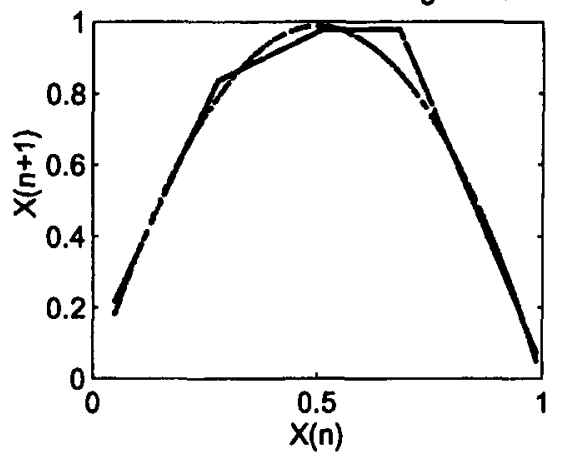

C. Trained Network using Combination

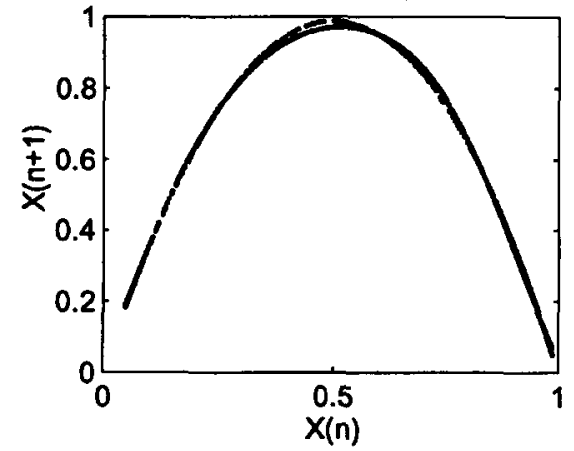

Figure 2. Results of training the network to predict the logistic function using three different transfer functions. A perfectly trained network should match the parabola. 
back propagation equations as Mpitsos and Burton (1992) for adjusting thresholds and weights.

A second training procedure was then implemented for the same network, but it was trained to predict the next value of the logistic equation

$$
X_{n+1}=k X_{n}\left(1-X_{n}\right)
$$

We tested the effect of both transfer functions with a chaotic mode $(k=3.95)$ in solving this task. Since a trained network will not generate a unique set of weights and thresholds for any given task, we retrained the network 1,000 times with different random starting values for weights and thresholds. This gave us 1,000 sets of weights and thresholds. Each of these 1,000 sets converged and resulted in an equally well-trained network.

\section{RESULTS}

Our tests show that there was a distinct difference in the behavior of the networks depending on the selection of transfer functions. The contrast between the two transfer functions on the identify function task can be seen in Figure 1. The network using a linear transfer function was able to obtain exact transmittance properties of this linear task (Figure 1D), while the sigmoid function yielded only an approximate result (Figure 1C).

Distinct differences were also evident when the network was trained to solve the more complex problem of predicting the $X_{n+1}$ value of the logistic equation. Again, the network could successfully be trained using either transfer function; however, the convergence properties were different and both functions left a large squared error. These results are illustrated in Figure 2, which plots the predicted $X_{n+1}$ value versus the entering $X_{n}$ value for each network trained. A perfectly trained network should follow the parabolic relationship of the logistic function.

As a post hoc exercise we used the sigmoid transfer function for the hidden neurons and the linear transfer function for the output neuron. The better fit to the distribution of the chaotic data resulting from the application of this more flexible arrangement is shown in Figure 2C. This strategy is somewhat common in the computer literature (Harvey, 1994) but has not been considered as a tool to make networks more neuromorphic.

The numerical values for weights $\left(w i_{\mathrm{i}}\right.$ and $\left.w o_{\mathrm{i}}\right)$ and thresholds are usually not reported in investigations of this type because a network will successfully converge with many possible sets of weights (Bremner, Gotts, \& Denham, 1994). The emphasis instead is placed on how well the network is trained and its learning rate. However, if there are similarities between the connectionist networks and the biological neurons, there must be some significance to the weights. Although there are too many dimensions even in a simple network to view all parameters on the same plot, the relationships of groups of two or three of these parameters exhibit interesting characteristics.

For example, Figure $3 \mathrm{~A}$ is a plot of over 1,000 sets of hidden-layer-to-input-layer weights that solved the simple identity task equally well. They form a spherical shape in 3-D space. When a slice of this shape is viewed from the top, we see that it is actually a spherical shell. Fig-

\section{A. Relationship of Input Weights}
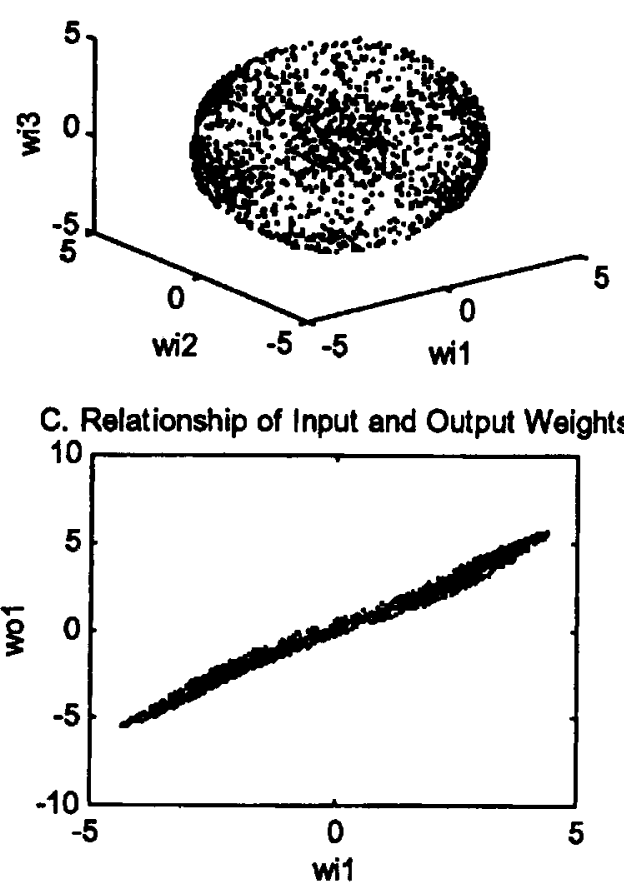

\section{B. Sliced view of A}

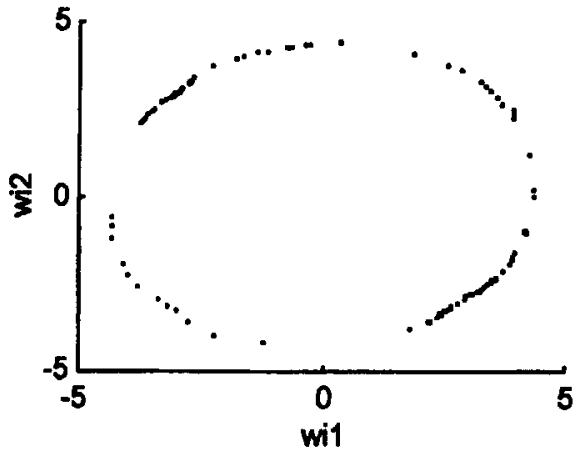

D. Relationship of Input Weight and Threshold

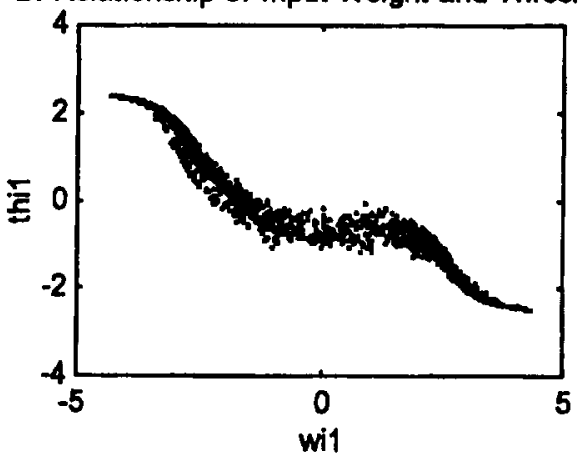

Figure 3. The relationship of thresholds and synaptic weights for the network trained to do the identity task with a chaotic input generated by the 3.95 logistic equation. 


\section{A. Relationship of Input Weights}
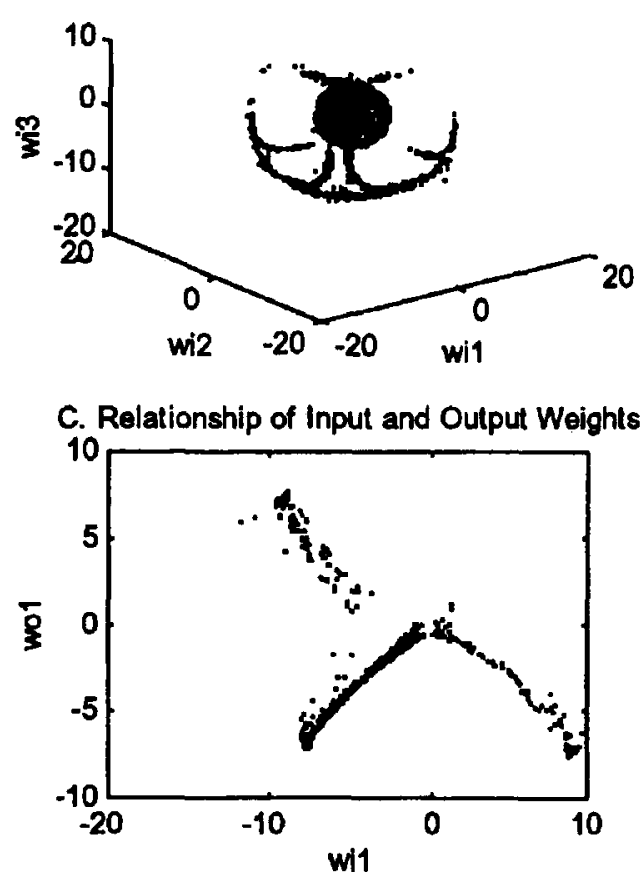

B. Top view of A

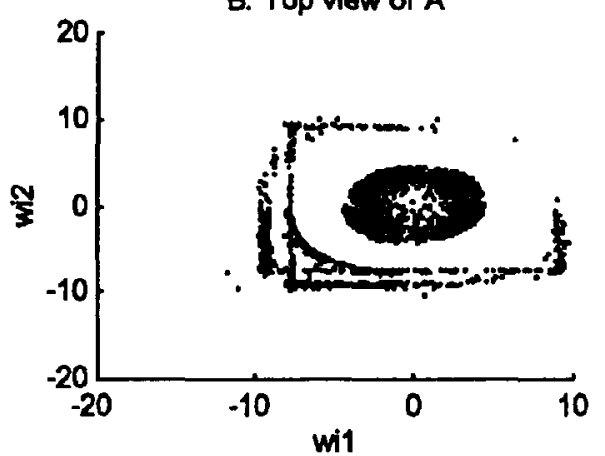

D. Relationship of Input Weight and Threshold

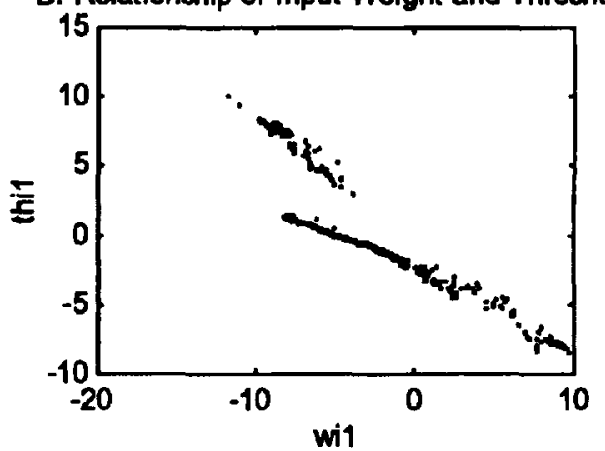

Figure 4. The relationship of the thresholds and synaptic weights for the network trained to predict the next value of a chaotic array generated by the 3.95 logistic equation.

ure $3 \mathrm{C}$ shows that the relationship between input and output weights of a hidden neuron is almost linear. Figure $3 \mathrm{D}$ is a plot of the range of input weights between the input neuron, and a hidden neuron compared to threshold values for the hidden neuron. Similar patterns were obtained for a network using the bounded linear transfer function trained on the predictive task.

The same graphic techniques were applied to the network trained to solve the more complicated prediction problem. The plots in Figure 4 show that the relationship of the weights has changed drastically. The range of input weights plotted in Figure 4A follows several straight and curved paths in 3-D space, but none intersect with the spherical shell of weights that solve the simpler identity function task. This is most evident when viewed from the top in Figure 4B. There is still a strong linear relationship between input and output weights associated with a hidden neuron, as shown in Figure 4C, but another weaker relationship also exists at the extremes. The relationship between threshold and input weight is strong for the hidden neuron (Figure 4D) at midrange, but weaker at the extremes.

\section{DISCUSSION}

Support for our original hypothesis that a linear transfer function would converge to better fit a linear identity function task than would a sigmoid transfer function is apparent from Figure 1. If Gardner's (1993) assertion that there is biological evidence for back propagation in bio- logical neurons is true, the simple examples illustrated here on a back propagation network can lead to further biological extension. For instance, we showed that either a linear or a sigmoid transfer function will allow a neural network to learn linear or nonlinear data, but the distribution of synaptic weights is different. We also found that using different but similar transfer functions for each layer can be beneficial, particularly when one is dealing with a chaotic signal (Figure 2C).

It is well known that there are several different types of neural transmitters involved in synapses (Clarke, 1989). These transmitters must exhibit slightly different physical diffusion properties along with different chemical interactions at the cell membranes. Thus, variations in characteristics probably exist among neurons. The differences in the transfer functions we used are solely dependent on how each function approaches the same maximum or minimum limiting value. Therefore, the transfer function affects the rate of change of the output of the simulated neurons and is at least a candidate for the biological ana$\log$ that controls rate of firing.

Extending this analogy further, the weights in the network can be related to firing rate. We tested a network that solved two problems using two similar transfer functions, but the domain of synaptic weights did not intersect. In fact, the possible weights that solved the more difficult problem (prediction) were larger, but occupied a relatively sparse (or restricted) area in 3-D space. A biological analog would be neurons that exhibit different firing rates and patterns to accomplish different tasks. In our 
examples, the predictive case led to the more active network. Negative weights, present in almost all our solution sets, might represent presynaptic signals that inhibit the firing rate. In short, further investigation of the relationships among transfer functions and synaptic weights will help us better understand the workings of biological neural networks.

\section{REFERENCES}

Bremner, F. J., \& Denham, D. L. (1993). Lotus 1-2-3 simulation of frequency encoding on the basilar membrane. Behavior Research Methods, Instruments, \& Computers, 25, 208-211.

Bremner, F. J., Gotts, S. J., \& Denham, D. L. (1994). Hinton diagrams: Viewing connection strengths in neural networks. Behavior Research Methods, Instruments, \& Computers, 26, 215-218.

Bremner, F. J., \& Yost, M. (1992). Assessing the simulation of chaotic systems. Behavior Research Methods, Instruments, \& Computers, 24, 336-339.

ClaRKE, K. A. (1989). Neurophysiology: Applications in the behavioral and biomedical sciences. Chichester, U.K.: Ellis Horwood.
GARDNER, D. (ED.) (1993). The neurobiology of neural networks. Cambridge, MA: MIT Press.

HaRVEY, R. L. (1994). Neural network principles. Englewood Cliffs, NJ: Prentice-Hall.

Hinton, G. E., \& ANDERson, J. A. (EDs.) (1981). Parallel models of associative memory. Hillsdale, $\mathrm{NJ}$ : Erlbaum.

LAWRENCE, J. (1993). Introduction to neural networks: Design, theory and applications. Nevada City, CA: California Scientific Software Press.

MACGREGOR, R. J. (1993). Theoretical mechanics of biological neural networks. San Diego: Academic Press.

MATLAB, Version 4.0 [Computer software] (1994). Natick, MA: Math Works.

Mpitsos, G., \& Burton, R. (1992). Convergence and divergence in neural networks: Processing of chaos and biological analogy. Neural Networks, 5, 605-625.

Mpitsos, G., Burton, R., \& Creech, C. (1988). Connectionist networks learn to transmit chaos. Brain Research Bulletin, 21, 539-546.

(Manuscript received January 9, 1996; revision accepted for publication January 30,1996 .) 